517.54

doi:10.13108/2018-10-1-80

UDC 517.54

\title{
BEHAVIOR OF SINGULAR INTEGRAL WITH HILBERT KERNEL AT WEAK CONTINUITY POINT OF DENSITY
}

\section{R.B. SALIMOV}

Abstract. We consider the singular integral with the Hilbert kernel

$$
I\left(\gamma_{0}\right)=\int_{0}^{2 \pi} \varphi(\gamma) \cot \frac{\gamma-\gamma_{0}}{2} d \gamma
$$

whose density $\varphi(\gamma)$ is a continuous in $[0,2 \pi]$ function, $\gamma_{0} \in[0,2 \pi], \varphi(0)=\varphi(2 \pi)$, and the integral is treated in the sense of its principal value. We assume that in the vicinity of a fixed point $\gamma=c, c \in\left(c^{-}, c^{+}\right) \subset[0,2 \pi], c^{+}-c^{-}<1$, the density $\varphi(\gamma)$ satisfies the representation $\varphi(\gamma)=\frac{\Phi(\gamma)}{\left(-\ln \sin ^{2} \frac{\gamma-c}{2}\right)^{\beta}}, \gamma \in\left(c^{-}, c^{+}\right)$, where $\Phi(\gamma)$ is a given continuous in $\left[c^{-}, c\right],\left[c, c^{+}\right]$ function with not necessarily coinciding one-sided limits $\Phi(c-0)$ and $\Phi(c+0), \beta$ is a given number, and $\beta>1$. We suppose that the representations $\Phi(\gamma)-\Phi(c \pm 0)=\frac{\chi(\gamma)}{\left(-\ln \sin ^{2} \frac{\gamma-c}{2}\right)^{\delta}}$, $\chi^{\prime}(\gamma)=\frac{\nu(\gamma)}{\left(-\ln \sin ^{2} \frac{\gamma-c}{2}\right) \tan \frac{\gamma-c}{2}}$, hold, where $\delta>0$ is a given number, $\chi(\gamma), \nu(\gamma)$ are given functions continuous in each of the intervals $\left[c^{-}, c\right],\left[c, c^{+}\right], \nu(c \pm 0)=0, \Phi(c+0)$ is taken as $\gamma>c, \Phi(c-0)$ is taken as $\gamma<c$.

We prove that under the above conditions the representation

$$
\begin{aligned}
& I\left(\gamma_{0}\right)-I(c)=\frac{\Phi(c-0)-\Phi(c+0)}{(\beta-1)\left(-\ln \sin ^{2} \frac{\gamma_{0}-c}{2}\right)^{\beta-1}} \\
& \quad-\frac{U(c+0)-U(c-0)}{\tilde{\beta}(\tilde{\beta}-1)\left(-\ln \sin ^{2} \frac{\gamma_{0}-c}{2}\right)^{\tilde{\beta}-1}}+o\left(\frac{1}{\left(-\ln \sin ^{2} \frac{\gamma_{0}-c}{2}\right)^{\tilde{\beta}-1}}\right)+O\left(\frac{1}{\left(-\ln \sin ^{2} \frac{\gamma_{0}-c}{2}\right)^{\beta}}\right),
\end{aligned}
$$

holds as $\gamma_{0} \rightarrow c$. Here $\tilde{\beta}=\beta+\delta, \beta>1, \delta>0, U(c+0)-U(c-0)=\tilde{\beta}(\chi(c+0)-\chi(c-0))$. We also consider the case $\beta=1$. A distinguishing feature of the paper is that while studying the behavior of the considered singular integral in the vicinity of the weak continuity point of its density, we need the Hölder condition no for the density neither for a component of the density. This feature allowed us to extend the range of possible applications of our results.

Keywords: singular integral, Hilbert kernel, Hölder condition, weak continuity.

Mathematics Subject Classification: $30 \mathrm{G} 12$

R.B. Salimov, Behavior of singular integral with Hilbert Kernel at Weak CONtinuity point OF DENSITY.

(c) SAlimov R.B. 2018.

The work is financially supported by RFBR (project no. 12-01-00636-a).

Submitted February 8, 2017. 


\section{Introduction. Formulation of Problem}

We consider a singular integral with a Hilbert kernel

$$
I\left(\gamma_{0}\right)=\int_{0}^{2 \pi} \varphi(\gamma) \cot \frac{\gamma-\gamma_{0}}{2} d \gamma
$$

assuming that the integral density $\varphi(\gamma)$ is a continuous function defined in the interval $[0,2 \pi]$, $\gamma_{0} \in[0,2 \pi], \varphi(0)=\varphi(2 \pi)$, and the integral is treated in the sense of the principal value.

We suppose that in the vicinity of a fixed point $\gamma=c, c \in\left(c^{-}, c^{+}\right) \subset[0,2 \pi], c^{+}-c^{-}<1$, the integral density $\varphi(\gamma)$ satisfies the representation

$$
\varphi(\gamma)=\frac{\Phi(\gamma)}{\left(-\ln \sin ^{2} \frac{\gamma-c}{2}\right)^{\beta}}, \quad \gamma \in\left(c^{-}, c^{+}\right)
$$

where $\Phi(\gamma)$ is a given function continuous in each of the intervals $\left[c^{-}, c\right],\left[c, c^{+}\right]$; in the general situation, the one-sided limits $\Phi(c-0), \Phi(c+0)$ do not coincide; $\beta$ is a given number and $\beta>1$.

Opposite to the conditions assumed in works [1], [2], here we suppose that the representations

$$
\begin{aligned}
& \Phi(\gamma)-\Phi(c \pm 0)=\frac{\chi(\gamma)}{\left(-\ln \sin ^{2} \frac{\gamma-c}{2}\right)^{\delta}}, \\
& \chi^{\prime}(\gamma)=\frac{\nu(\gamma)}{\left(-\ln \sin ^{2} \frac{\gamma-c}{2}\right) \tan \frac{\gamma-c}{2}}
\end{aligned}
$$

hold, where $\delta>0$ is a given number, $\chi(\gamma), \nu(\gamma)$ are given functions continuous in each of the intervals $\left[c^{-}, c\right],\left[c, c^{+}\right], \nu(c \pm 0)=0$. In formula (3) and in what follows the sign ' + ' is chosen for $\gamma>c$ and the sign '-' corresponds to $\gamma<c$.

In view of (3), we write formula (2) as

$$
\varphi(\gamma)=\frac{\chi(\gamma)}{\left(-\ln \sin ^{2} \frac{\gamma-c}{2}\right)^{\beta+\delta}}+\frac{\Phi(c \pm 0)}{\left(-\ln \sin ^{2} \frac{\gamma-c}{2}\right)^{\beta}}, \gamma \in\left(c^{-}, c^{+}\right)
$$

\section{MAIN RESUlts. I}

Assuming for simplicity $c^{-}>0, c^{+}<2 \pi$, we represent integral (1) as

$$
\begin{aligned}
I\left(\gamma_{0}\right)= & \left(\int_{0}^{c^{-}}+\int_{c^{+}}^{2 \pi} \varphi(\gamma) \cot \frac{\gamma-\gamma_{0}}{2} d \gamma+\int_{c^{-}}^{c^{+}} \frac{\chi(\gamma)}{\left(-\ln \sin ^{2} \frac{\gamma-c}{2}\right)^{\beta+\delta}} \cot \frac{\gamma-\gamma_{0}}{2} d \gamma\right. \\
& +\int_{c^{-}}^{c^{+}} \frac{\Phi(c \pm 0)}{\left(-\ln \sin ^{2} \frac{\gamma-c}{2}\right)^{\beta}} \cot \frac{\gamma-\gamma_{0}}{2} d \gamma=J_{1}\left(\gamma_{0}\right)+J_{2}\left(\gamma_{0}\right)+J_{3}\left(\gamma_{0}\right)+J_{4}\left(\gamma_{0}\right), \quad \gamma_{0} \neq c .
\end{aligned}
$$

The integrals $J_{1}\left(\gamma_{0}\right), J_{2}\left(\gamma_{0}\right)$ are differentiable in each interior point $\gamma_{0}$ of the interval $\left(c^{-}, c^{+}\right)$. The integral densities $J_{3}\left(\gamma_{0}\right), J_{4}\left(\gamma_{0}\right)$ are differentiable in the interval $\left[c^{-}, c^{+}\right]$excluding the point $c$. This is why the integrals $J_{3}\left(\gamma_{0}\right), J_{4}\left(\gamma_{0}\right)$ satisfy the Hölder condition, shortly $H$ condition, in each closed interval located inside $\left[c^{-}, c\right]$ or $\left[c, c^{+}\right]$, see [3].

In what follows, to reduce the writing, we employ the shorthand notation

$$
\operatorname{ls} \gamma=-\ln \sin ^{2} \gamma
$$


The right hand side of the formula

$$
\left(\frac{1}{\left(\operatorname{ls} \frac{\gamma-c}{2}\right)^{\beta}}\right)_{\gamma}^{\prime}=\frac{\beta}{\left(\operatorname{ls} \frac{\gamma-c}{2}\right)^{\beta+1} \tan \frac{\gamma-c}{2}}
$$

is a function decreasing in the interval $\left(c-2 e^{-\beta-1}, c+2 e^{-\beta-1}\right)$ since

$$
\left[\left(\operatorname{ls} \frac{\gamma-c}{2}\right)^{\beta+1} \tan \frac{\gamma-c}{2}\right]_{\gamma}^{\prime}>0
$$

in each of the intervals $c<\gamma<c+2 e^{-\beta-1}, c-2 e^{-\beta-1}<\gamma<c$. Indeed, as $0<\gamma-c<1$, we observe that $\sin x<x$ as $x>0$ and we obtain

$$
\left[\left(\operatorname{ls} \frac{\gamma-c}{2}\right)^{\beta+1} \tan \frac{\gamma-c}{2}\right]_{\gamma}^{\prime}>\left(\operatorname{ls} \frac{\gamma-c}{2}\right)^{\beta}\left(-\beta-1+\ln \frac{2}{\gamma-c}\right)>0 .
$$

Here the sum in the brackets in the right hand side vanishes as $\gamma=c+2 e^{-\beta-1}$ being positive for $c<\gamma<c+2 e^{-\beta-1}$. Therefore, as $c<\gamma<c+2 e^{-\beta-1}$, inequality (8) holds. It is easy to check that the latter inequality holds also for $\gamma \in\left(c-2 e^{-\beta-1}, c\right)$.

In what follows we assume that

$$
c^{-}=c-2 e^{-\beta-1}, \quad c^{+}=c+2 e^{-\beta-1}
$$

in formula (6).

Integrating by parts, we represent the term $J_{4}\left(\gamma_{0}\right)$ in formula (6) as

$$
J_{4}\left(\gamma_{0}\right)=-\frac{\Phi(c+0)}{\left(\operatorname{ls} \frac{c^{+}-c}{2}\right)^{\beta}} \operatorname{ls} \frac{c^{+}-\gamma_{0}}{2}+\frac{\Phi(c-0)}{\left(\operatorname{ls} \frac{c^{-}-c}{2}\right)^{\beta}} \operatorname{ls} \frac{c^{-}-\gamma_{0}}{2}+\beta K\left(\gamma_{0}\right),
$$

where

$$
K\left(\gamma_{0}\right)=\int_{c^{-}}^{c^{+}} \frac{\Phi(c \pm 0)}{\left(\operatorname{ls} \frac{\gamma-c}{2}\right)^{\beta+1} \tan \frac{\gamma-c}{2}}\left(\operatorname{ls} \frac{\gamma-\gamma_{0}}{2}\right) d \gamma
$$

The first two terms in the right hand side of formula (10) are differentiable in each interior point of the interval $\left(c^{-}, c^{+}\right)$. Therefore, we need to study the behavior of the function $K\left(\gamma_{0}\right)$ as $\gamma_{0} \rightarrow c$.

We consider the case $\gamma_{0}>c$. We assume that $\gamma_{0}<c+e^{-\beta-1}$ taking into consideration that the difference $\gamma_{0}-c$ is assumed to be small. Then by (9) we have

$$
c<\gamma_{0}<2 \gamma_{0}-c<c+2 e^{-\beta-1}=c^{+} \text {. }
$$

Denoting

$$
c_{\gamma_{0}}^{-}=\gamma_{0}-2 e^{-\beta-1},
$$

for $c<\gamma_{0}<c+e^{-\beta-1}$ we get $c^{-}<c_{\gamma_{0}}^{-}=\gamma_{0}-2 e^{-\beta-1}<2 c-\gamma_{0}<c$.

We write formula (11) as

$$
\begin{aligned}
K\left(\gamma_{0}\right) & =\left(\int_{c^{-}}^{c_{\gamma_{0}}^{-}}+\int_{c_{\gamma_{0}}^{-}}^{2 c-\gamma_{0}}+\int_{2 c-\gamma_{0}}^{c}+\int_{c}^{\frac{c+\gamma_{0}}{2}}+\int_{\frac{c+\gamma_{0}}{2}}^{2 \gamma_{0}-c}+\int_{2 \gamma_{0}-c}^{c^{+}}\right) \frac{\Phi(c \pm 0)\left(\operatorname{ls} \frac{\gamma-\gamma_{0}}{2}\right)}{\left(\operatorname{ls} \frac{\gamma-c}{2}\right)^{\beta+1} \tan \frac{\gamma-c}{2}} d \gamma \\
& =\Phi(c-0)\left[K_{1}\left(\gamma_{0}\right)+K_{2}\left(\gamma_{0}\right)+K_{3}\left(\gamma_{0}\right)\right]+\Phi(c+0)\left[K_{4}\left(\gamma_{0}\right)+K_{5}\left(\gamma_{0}\right)+K_{6}\left(\gamma_{0}\right)\right] .
\end{aligned}
$$

The integrand for $K_{4}\left(\gamma_{0}\right)$ is a function positive as $\gamma \in\left(c, \frac{c+\gamma_{0}}{2}\right)$, its factor $\operatorname{ls}_{\mathrm{s}} \frac{\gamma-\gamma_{0}}{2}$ is a continuous increasing positive function in the integration interval. 
This is why, replacing the factor $\mathrm{ls}_{\mathrm{s}} \frac{\gamma-\gamma_{0}}{2}$ by its minimum in the formula for $K_{4}\left(\gamma_{0}\right)$ and replacing ls $\frac{c-\gamma_{0}}{2}$, ls $\frac{c-\gamma_{0}}{4}$ by their maxima, we obtain two integrals which can be calculated due to (7) and which bound the integral $K_{4}\left(\gamma_{0}\right)$ from above and below, that is, for the values of the mentioned integrals we obtain the inequalities

which are equivalent to

$$
\frac{\operatorname{ls} \frac{\gamma_{0}-c}{2}}{\beta\left(\operatorname{ls} \frac{\gamma_{0}-c}{4}\right)^{\beta}}<K_{4}\left(\gamma_{0}\right)<\frac{\operatorname{ls} \frac{\gamma_{0}-c}{4}}{\beta\left(\operatorname{ls} \frac{\gamma_{0}-c}{4}\right)^{\beta}},
$$

$$
\frac{\ln \left(4 \cos ^{2} \frac{\gamma_{0}-c}{4}\right)}{\beta\left(\operatorname{ls} \frac{\gamma_{0}-c}{4}\right)^{\beta}}>-K_{4}\left(\gamma_{0}\right)+\frac{1}{\beta\left(\operatorname{ls} \frac{\gamma_{0}-c}{4}\right)^{\beta-1}}>0 .
$$

Therefore, for the values $\gamma_{0}$ close to $c$, we have

$$
-K_{4}\left(\gamma_{0}\right)+\frac{1}{\beta\left(\operatorname{ls} \frac{\gamma_{0}-c}{4}\right)^{\beta-1}}=O\left(1 /\left(\operatorname{ls} \frac{\gamma_{0}-c}{4}\right)^{\beta}\right) .
$$

Hereinafter, as usually, by $O(\eta)$ we mean a quantity, for which the absolute value of the fraction $O(\eta) / \eta$ is bounded for all sufficiently small (sufficiently large) absolute values of $\eta$.

In the first part of the latter formula, instead of $O\left(1 /\left(\operatorname{ls}_{\mathrm{s}} \frac{\gamma_{0}-c}{4}\right)^{\beta}\right)$, we can take $O\left(1 /\left(\operatorname{ls} \frac{\gamma_{0}-c}{2}\right)^{\beta}\right)$, since $-\mathrm{ls} \frac{\gamma_{0}-c}{2} \sim-\operatorname{ls} \frac{\gamma_{0}-c}{4}$ as $\gamma_{0} \rightarrow c$, and moreover,

$$
1 /\left(\operatorname{ls} \frac{\gamma_{0}-c}{4}\right)^{\beta-1}=1 /\left(\operatorname{ls} \frac{\gamma_{0}-c}{2}\right)^{\beta-1}+O\left(1 /\left(\operatorname{ls} \frac{\gamma_{0}-c}{2}\right)^{\beta}\right), \quad \gamma_{0} \rightarrow c .
$$

Indeed,

$$
\begin{aligned}
& \frac{1}{\left(\operatorname{ls} \frac{\gamma_{0}-c}{4}\right)^{\beta-1}}-\frac{1}{\left(\operatorname{ls} \frac{\gamma_{0}-c}{2}\right)^{\beta-1}} \\
& =\frac{1}{\left(\operatorname{ls} \frac{\gamma_{0}-c}{2}\right)^{\beta-1}}\left[\left(1+\frac{\ln \left(2 \cos \frac{\gamma_{0}-c}{4}\right)^{2}}{\operatorname{ls} \frac{\gamma_{0}-c}{2}}\right)^{1-\beta}-1\right]=O\left(1 /\left(\operatorname{ls} \frac{\gamma_{0}-c}{2}\right)^{\beta}\right), \quad \gamma_{0} \rightarrow c .
\end{aligned}
$$

Thus, we arrive at the formula

$$
K_{4}\left(\gamma_{0}\right)=\frac{1}{\beta\left(\operatorname{ls} \frac{\gamma_{0}-c}{2}\right)^{\beta-1}}+O\left(1 /\left(\operatorname{ls} \frac{\gamma_{0}-c}{2}\right)^{\beta}\right), \quad \gamma_{0} \rightarrow c .
$$

Since according (8), the factor $1 /\left[\left(\operatorname{ls} \frac{\gamma-c}{2}\right)^{\beta+1} \tan \frac{\gamma-c}{2}\right]$ decreases in the integration interval for $K_{5}\left(\gamma_{0}\right)$, then

$$
0<K_{5}\left(\gamma_{0}\right)<\frac{1}{\left(\operatorname{ls} \frac{\gamma_{0}-c}{4}\right)^{\beta+1} \tan \frac{\gamma_{0}-c}{4}} \int_{\frac{c+\gamma_{0}}{2}}^{2 \gamma_{0}-c} \operatorname{ls} \frac{\gamma-\gamma_{0}}{2} d \gamma
$$

The integral in this formula can be represented as

$$
\int_{\frac{c+\gamma_{0}}{2}}^{2 \gamma_{0}-c} \operatorname{ls} \frac{\gamma-\gamma_{0}}{2} d \gamma=\left.\left(\gamma-\gamma_{0}\right) \operatorname{ls} \frac{\gamma-\gamma_{0}}{2}\right|_{\frac{c+\gamma_{0}}{2}} ^{2 \gamma_{0}-c}+\int_{\frac{c+\gamma_{0}}{2}}^{2 \gamma_{0}-c}\left(\gamma-\gamma_{0}\right) \cot \frac{\gamma-\gamma_{0}}{2} d \gamma .
$$

Substituting this expression into formula $(16)$ and taking into consideration that

$$
0<\int_{\frac{c+\gamma_{0}}{2}}^{2 \gamma_{0}-c}\left(\gamma-\gamma_{0}\right) \cot \frac{\gamma-\gamma_{0}}{2} d \gamma<d\left(\gamma_{0}-c\right), \quad d=\text { const }
$$


we obtain

$$
K_{5}\left(\gamma_{0}\right)=O\left(1 /\left(\operatorname{ls} \frac{\gamma_{0}-c}{2}\right)^{\beta}\right), \quad \gamma_{0} \rightarrow c .
$$

We represent the integral $K_{6}\left(\gamma_{0}\right)$ in formula (13) as

$$
K_{6}\left(\gamma_{0}\right)=\frac{1 /(\beta-1)}{\left(\operatorname{ls} \frac{c^{+}-\gamma_{0}}{2}\right)^{\beta-1}}-\frac{1 /(\beta-1)}{\left(\operatorname{ls} \frac{\gamma_{0}-c}{2}\right)^{\beta-1}}-E_{6}\left(\gamma_{0}\right),
$$

where

$$
\begin{aligned}
& E_{6}\left(\gamma_{0}\right)=\int_{2 \gamma_{0}-c}^{c^{+}} A\left(\gamma, \gamma_{0}, c\right) \operatorname{ls} \frac{\gamma-\gamma_{0}}{2} d \gamma \\
& A\left(\gamma, \gamma_{0}, c\right)=1 /\left[\operatorname{ls} \frac{c-\gamma}{2}\right]^{\beta+1} \tan \frac{c-\gamma}{2}-1 /\left[\operatorname{ls} \frac{\gamma_{0}-\gamma}{2}\right]^{\beta+1} \tan \frac{\gamma_{0}-\gamma}{2} .
\end{aligned}
$$

It is easy to show that for each $\gamma_{0} \in\left(2 \gamma_{0}-c, c^{+}\right)$the inequality

$$
A\left(\gamma, \gamma_{0}, c\right)>0
$$

holds. Indeed, by (8) we have

$$
\left[\left(\operatorname{ls} \frac{\xi-\gamma}{2}\right)^{\beta+1} \tan \frac{\xi-\gamma}{2}\right]_{\xi}^{\prime}>0
$$

in the interval $\gamma-2 e^{-\beta-1}<\xi<\gamma$.

Let us consider the set of the latter intervals for $\gamma \in\left(2 \gamma_{0}-c, c^{+}\right)$. In this case we have $\gamma-2 e^{-\beta-1}<c$, since $\gamma<c+2 e^{-\beta-1}=c^{+}$, and $\gamma>\gamma_{0}$ since $\gamma>2 \gamma_{0}-c>\gamma_{0}$. Therefore, as $\gamma \in\left(2 \gamma_{0}-c, c^{+}\right)$, the interval $\gamma-2 e^{-\beta-1}<\xi<\gamma$ contains the interval $\left(c, \gamma_{0}\right)$. This is why for each $\gamma \in\left(2 \gamma_{0}-c, c^{+}\right)$, condition (22) holds in the interval $c<\xi<\gamma_{0}$. This means that in the latter interval the function $1 /\left[\operatorname{ls} \frac{\xi-\gamma}{2} \tan \frac{\xi-\gamma}{2}\right]$ decreases. This is why, according (20), inequality (21) holds true.

Observing that in the interval $2 \gamma_{0}-c<\gamma<c^{+}$the function $\mathrm{ls}_{\mathrm{s}} \frac{\gamma-\gamma_{0}}{2}$ decreases and is positive, by $(19)$ we get

$$
0<E_{6}\left(\gamma_{0}\right)<\operatorname{ls} \frac{\gamma_{0}-c}{2} \cdot \int_{2 \gamma_{0}-c}^{c^{+}} A\left(\gamma, \gamma_{0}, c\right) d \gamma .
$$

Calculating the integral in the right hand side, in view of (7) and (20) we arrive at the inequalities

$$
\begin{aligned}
0<E_{6}\left(\gamma_{0}\right)< & \frac{1}{\beta}\left(\operatorname{ls} \frac{\gamma_{0}-c}{2}\right)\left[\frac{1}{\left(\operatorname{ls}\left(\frac{c-\gamma_{0}}{2}+e^{-\beta-1}\right)\right)^{\beta}}-\frac{1}{\left(\operatorname{ls} e^{-\beta-1}\right)^{\beta}}\right] \\
& +\frac{1}{\beta\left(\operatorname{ls} \frac{\gamma_{0}-c}{2}\right)^{\beta-1}}\left[\frac{\left(\operatorname{ls} \frac{\gamma_{0}-c}{2}\right)^{\beta}}{\left(\operatorname{ls}\left(\gamma_{0}-c\right)\right)^{\beta}}-1\right] .
\end{aligned}
$$

The first difference in square brackets in the right hand side of the latter formula is an infinitesimal of order $\left(\gamma_{0}-c\right)$ as $\gamma_{0} \rightarrow c$, as an increment of a differentiable function. The second difference of the considered formula is equal to

$$
\left(1+\frac{-\ln \left(2 \cos \frac{\gamma_{0}-c}{2}\right)^{2}}{\operatorname{ls} \frac{\gamma_{0}-c}{2}}\right)^{-\beta}-1
$$

and is an infinitesimal of order $1 /\left(\operatorname{ls} \frac{\gamma_{0}-c}{2}\right)$ as $\gamma_{0} \rightarrow c$. 
This is why we obtain

$$
E_{6}\left(\gamma_{0}\right)=O\left(1 /\left(\operatorname{ls} \frac{\gamma_{0}-c}{2}\right)^{\beta}\right), \quad \gamma_{0} \rightarrow c .
$$

Taking into consideration the latter relation, we represent formula (18) as

$$
K_{6}\left(\gamma_{0}\right)=\frac{1 /(\beta-1)}{\left(\operatorname{ls} \frac{c^{+}-c}{2}\right)^{\beta-1}}-\frac{1 /(\beta-1)}{\left(\operatorname{ls} \frac{\gamma_{0}-c}{2}\right)^{\beta-1}}+O\left(1 /\left(\operatorname{ls} \frac{\gamma_{0}-c}{2}\right)^{\beta}\right), \quad \gamma_{0} \rightarrow c .
$$

Here we have employed that the difference between the first terms in the right hand sides of formulae (18), 25) is an infinitesimal of order $\left(\gamma_{0}-c\right)$ as $\gamma_{0} \rightarrow c$.

We assume that $-K_{3}\left(\gamma_{0}\right)$ is an integral of a positive function containing the factor tan $\frac{c-\gamma}{2}$ in the denominator. Since the factor $\mathrm{ls}_{\mathrm{s}} \frac{\gamma-\gamma_{0}}{2}$ in the integrand in $-K_{3}\left(\gamma_{0}\right)$ is an increasing function in the integration interval with the minimum $\mathrm{ls}\left(\gamma_{0}-c\right)$ and the maximum $\mathrm{l}_{\mathrm{s}} \frac{\gamma_{0}-c}{2}$ we proceed as in studying the integral $K_{4}\left(\gamma_{0}\right)$ to arrive to inequalities

$$
\frac{\operatorname{ls}\left(\gamma_{0}-c\right)}{\beta\left(\operatorname{ls} \frac{\gamma_{0}-c}{2}\right)^{\beta}}<-K_{3}\left(\gamma_{0}\right)<\frac{\operatorname{ls} \frac{\gamma_{0}-c}{2}}{\beta\left(\operatorname{ls} \frac{\gamma_{0}-c}{2}\right)^{\beta}},
$$

which is equivalent to

$$
\frac{\ln \left(4 \cos ^{2} \frac{\gamma_{0}-c}{2}\right)}{\beta\left(\operatorname{ls} \frac{\gamma_{0}-c}{2}\right)^{\beta}}>K_{3}\left(\gamma_{0}\right)+\frac{1}{\beta\left(\operatorname{ls} \frac{\gamma_{0}-c}{2}\right)^{\beta-1}}>0 .
$$

By this we see that

$$
K_{3}\left(\gamma_{0}\right)+\frac{1}{\beta\left(\operatorname{ls} \frac{\gamma_{0}-c}{2}\right)^{\beta-1}}=O\left(\frac{1}{\left(\operatorname{ls} \frac{\gamma_{0}-c}{2}\right)^{\beta}}\right), \quad \gamma_{0} \rightarrow c .
$$

Similar to formula $(18)$, we have

$$
K_{2}\left(\gamma_{0}\right)=\frac{1 /(\beta-1)}{\left(\operatorname{ls}\left(c-\gamma_{0}\right)\right)^{\beta-1}}-\frac{1 /(\beta-1)}{\left(\operatorname{ls} e^{-\beta-1}\right)^{\beta-1}}-E_{2}\left(\gamma_{0}\right),
$$

where

$$
E_{2}\left(\gamma_{0}\right)=\int_{c_{\gamma_{0}}^{-}}^{2 c-\gamma_{0}} A\left(\gamma, \gamma_{0}, c\right) \operatorname{ls} \frac{\gamma-\gamma_{0}}{2} d \gamma
$$

$A\left(\gamma, \gamma_{0}, c\right)$ is the function defined by formula $(20)$.

As $\gamma \in\left(c_{\gamma_{0}}^{-}, 2 c-\gamma_{0}\right)$, the interval $\gamma<\xi<\gamma+2 e^{-\beta-1}$ contains the interval $\left(c, \gamma_{0}\right)$ since here we have $\gamma<2 c-\gamma_{0}<c, \gamma+2 e^{-\beta-1}>\gamma_{0}$ because $\gamma>c_{\gamma_{0}}^{-}=\gamma_{0}-2 e^{-\beta-1}$. Therefore, for each $\gamma \in\left(c_{\gamma_{0}}^{-}, 2 c-\gamma_{0}\right)$, condition 22 holds in the interval $c<\xi<\gamma_{0}$ and this is why we have the inequality $A\left(\gamma, \gamma_{0}, c\right)>0$. Taking into consideration the latter and observing that $\operatorname{ls} \frac{\gamma-\gamma_{0}}{2}$ is an increasing positive function in the interval $c_{\gamma_{0}}^{-}<\gamma<2 c-\gamma_{0}$, according 28) we have

$$
0<E_{2}\left(\gamma_{0}\right)<\operatorname{ls}\left(\gamma_{0}-c\right) \cdot \int_{c_{\gamma_{0}}^{-}}^{2 c-\gamma_{0}} A\left(\gamma, \gamma_{0}, c\right) d \gamma
$$


Calculating the integral in this formula, we obtain

$$
\begin{aligned}
0<E_{2}\left(\gamma_{0}\right)< & \frac{1}{\beta} \operatorname{ls}\left(\gamma_{0}-c\right) \cdot\left[\frac{1}{\left(\operatorname{ls}\left(-\frac{\gamma_{0}-c}{2}+e^{-\beta-1}\right)\right)^{\beta}}-\frac{1}{\left(\operatorname{ls}\left(e^{-\beta-1}\right)\right)^{\beta}}\right] \\
& +\frac{1}{\beta\left(\operatorname{ls}\left(\gamma_{0}-c\right)\right)^{\beta-1}} \cdot\left[1-\frac{\left(\operatorname{ls}\left(\gamma_{0}-c\right)\right)^{\beta}}{\left(\operatorname{ls} \frac{\gamma_{0}-c}{2}\right)^{\beta}}\right] .
\end{aligned}
$$

The first difference in square brackets in the right hand of this formula is the same as in inequality (23), while the second term (product) in the right hand side of the latter formula differs from the corresponding term (product) in the right hand side in 25) only by the factor $-\operatorname{ls}\left(\gamma_{0}-c\right) / \operatorname{ls} \frac{\gamma_{0}-c}{2}$ bounded as $\gamma_{0} \rightarrow c$. Therefore,

$$
E_{2}\left(\gamma_{0}\right)=O\left(1 /\left(\operatorname{ls} \frac{\gamma_{0}-c}{2}\right)^{\beta}\right), \quad \gamma_{0} \rightarrow c .
$$

We observe that similar to formula (14), for small $\gamma_{0}-c$ we have

$$
\frac{1}{\left(\operatorname{ls}\left(\gamma_{0}-c\right)\right)^{\beta-1}}-\frac{1}{\left(\operatorname{ls} \frac{\gamma_{0}-c}{2}\right)^{\beta-1}}=O\left(1 /\left(\operatorname{ls} \frac{\gamma_{0}-c}{2}\right)^{\beta}\right) \text {. }
$$

We write representation (27) as

$$
K_{2}\left(\gamma_{0}\right)=\frac{1 /(\beta-1)}{\left(\operatorname{ls} \frac{\gamma_{0}-c}{2}\right)^{\beta-1}}-\frac{1 /(\beta-1)}{\left(\operatorname{ls} e^{-\beta-1}\right)^{\beta-1}}+O\left(1 /\left(\operatorname{ls} \frac{\gamma_{0}-c}{2}\right)^{\beta}\right), \quad \gamma_{0} \rightarrow c .
$$

Taking into consideration that in the interval $c^{-}<\gamma<c_{\gamma_{0}}^{-}$the function $\mathrm{ls} \frac{\gamma-\gamma_{0}}{2}$ increases and is positive, we get

$$
0<-K_{1}\left(\gamma_{0}\right)<\operatorname{ls}\left(e^{-\beta-1}\right) \cdot \int_{c^{-}}^{c_{\gamma_{0}}^{-}} \frac{d \gamma}{\left(\operatorname{ls} \frac{\gamma-c}{2}\right)^{\beta+1} \tan \frac{c-\gamma}{2}}=\left.\operatorname{ls}\left(e^{-\beta-1}\right) \frac{-1}{\beta\left(\operatorname{ls} \frac{\gamma-c}{2}\right)^{\beta}}\right|_{c^{-}} ^{c_{\gamma_{0}}^{-}}
$$

Hence, we obtain

$$
K_{1}\left(\gamma_{0}\right)=O\left(\gamma_{0}-c\right), \quad \gamma_{0} \rightarrow c .
$$

We substitute the obtained for $K_{j}\left(\gamma_{0}\right), j=\overline{1,6}$, expressions into formula (13) and we arrive at the following representation

$$
\begin{aligned}
K\left(\gamma_{0}\right)= & \frac{\Phi(c+0)-\Phi(c-0)}{(\beta-1)\left(\operatorname{ls}\left(e^{-\beta-1}\right)\right)^{\beta-1}}+\frac{\Phi(c-0)-\Phi(c+0)}{\beta(\beta-1)\left(\operatorname{ls} \frac{\gamma_{0}-c}{2}\right)^{\beta-1}} \\
& +O\left(1 /\left(\operatorname{ls} \frac{\gamma_{0}-c}{2}\right)^{\beta}\right), \quad \gamma_{0} \rightarrow c .
\end{aligned}
$$

Formulae (10), 11), 32 hold both in the case $\gamma_{0}=c$. In particular, the first term in the right hand side of formula (32) is equal to $K(c)$.

Employing formula (10), we write the expression for $J_{4}\left(\gamma_{0}\right)-J_{4}(c)$ and we take into consideration that

$$
-\operatorname{ls} \frac{c^{+}-\gamma_{0}}{2}+\operatorname{ls} \frac{c^{+}-c}{2}=O\left(\gamma_{0}-c\right)
$$

the difference in this formula is an infinitesimal of order $\left(\gamma_{0}-c\right)$ as $\gamma_{0} \rightarrow c$ because of the differentiability of the function $-\mathrm{ls}_{\mathrm{s}} \frac{c^{+}-\gamma_{0}}{2}$ at the point $c$ and there holds a similar identity obtained by replacing $c^{+}$by $c^{-}$.

Hence, taking into consideration $(32)$, we obtain

$$
J_{4}\left(\gamma_{0}\right)-J_{4}(c)=\frac{\Phi(c-0)-\Phi(c+0)}{(\beta-1)\left(\operatorname{ls} \frac{\gamma_{0}-c}{2}\right)^{\beta-1}}+O\left(1 /\left(\operatorname{ls} \frac{\gamma_{0}-c}{2}\right)^{\beta}\right), \quad \gamma_{0} \rightarrow c .
$$


We also observe that similar to (33), for the integrals $J_{1}\left(\gamma_{0}\right), J_{2}\left(\gamma_{0}\right)$ in formula (6) we have

$$
J_{1}\left(\gamma_{0}\right)-J_{1}(c)=O\left(\gamma_{0}-c\right), \quad J_{2}\left(\gamma_{0}\right)-J_{2}(c)=O\left(\gamma_{0}-c\right) .
$$

\section{MAIN RESULTS. II}

In the expression for the integral $J_{3}\left(\gamma_{0}\right)$ in formula (6), we denote $\tilde{\beta}=\beta+\delta$ and by formulae similar to (9), (12) we define

$$
\tilde{c}^{-}=c-2 e^{-\tilde{\beta}-1}, \quad \tilde{c}^{+}=c+2 e^{-\tilde{\beta}-1}, \quad \tilde{c}_{\gamma_{0}}^{-}=\gamma_{0}-2 e^{-\tilde{\beta}-1},
$$

and at that we have

$$
\tilde{c}^{-}>c^{-}, \quad \tilde{c}^{+}<c^{+}, \quad \tilde{c}_{\gamma_{0}}^{-}>c_{\gamma_{0}}^{-} .
$$

Moreover, we assume that $c<\gamma_{0}<c+e^{-\tilde{\beta}-1}$.

We represent the integral $J_{3}\left(\gamma_{0}\right)$ as

$$
J_{3}\left(\gamma_{0}\right)=\left(\int_{c^{-}}^{\tilde{c}^{-}}+\int_{\tilde{c}^{-}}^{\tilde{c}^{+}}+\int_{\tilde{c}^{+}}^{c^{+}}\right) \frac{\chi(\gamma)}{\left(\operatorname{ls} \frac{\gamma-c}{2}\right)^{\tilde{\beta}}} \cot \frac{\gamma-\gamma_{0}}{2} d \gamma=J_{31}\left(\gamma_{0}\right)+J_{32}\left(\gamma_{0}\right)+J_{33}\left(\gamma_{0}\right) .
$$

The integrals $J_{31}\left(\gamma_{0}\right), J_{33}\left(\gamma_{0}\right)$ are differentiable at each interior point $\gamma_{0}$ of the interval $\left(\tilde{c}^{-}, \tilde{c}^{+}\right)$. Integrating by parts, we write the integral $J_{32}\left(\gamma_{0}\right)$ as

$$
J_{32}\left(\gamma_{0}\right)=-\frac{\chi\left(\tilde{c}^{+}\right)}{\left(\operatorname{ls} \frac{\tilde{c}^{+}-c}{2}\right)^{\tilde{\beta}}} \operatorname{ls} \frac{\tilde{c}^{+}-\gamma_{0}}{2}+\frac{\chi\left(\tilde{c}^{-}\right)}{\left(\operatorname{ls} \frac{\tilde{c}^{-}-c}{2}\right)^{\tilde{\beta}}} \operatorname{ls} \frac{\tilde{c}^{-}-\gamma_{0}}{2}+L\left(\gamma_{0}\right),
$$

where

$$
L\left(\gamma_{0}\right)=\int_{\tilde{c}^{-}}^{\tilde{c}^{+}} \frac{U(\gamma)}{\left(\operatorname{ls} \frac{\gamma-c}{2}\right)^{\tilde{\beta}+1} \tan \frac{\gamma-c}{2}} \cdot \operatorname{ls} \frac{\gamma-\gamma_{0}}{2} d \gamma
$$

$U(\gamma)=\nu(\gamma)+\tilde{\beta} \chi(\gamma)$ is a function continuous in each of the intervals $\left[c^{-}, c\right],\left[c, c^{+}\right]$according formulae (3), (4).

Let $\tilde{K}_{j}\left(\gamma_{0}\right)$ be the integral obtained by the formula for $K_{j}\left(\gamma_{0}\right)$ in representation (13) as $j=\overline{1,6}$ via replacing $K, c^{-}, c_{\gamma_{0}}^{-}, c^{+}, \beta$ respectively by $\tilde{K}, \tilde{c}^{-}, \tilde{c}_{\gamma_{0}}^{-}, \tilde{c}^{+}, \tilde{\beta}$.

We write integral $(38)$ as

$$
L\left(\gamma_{0}\right)=L_{1}\left(\gamma_{0}\right)+L_{2}\left(\gamma_{0}\right)+\cdots+L_{6}\left(\gamma_{0}\right),
$$

where the terms in the right hand side are the integral taken respectively over the intervals

$$
\left(\tilde{c}^{-}, \tilde{c}_{\gamma_{0}}^{-}\right), \quad\left(\tilde{c}_{\gamma_{0}}^{-}, 2 c-\gamma_{0}\right), \quad\left(2 c-\gamma_{0}, c\right), \quad\left(c, \frac{c+\gamma_{0}}{2}\right), \quad\left(\frac{c+\gamma_{0}}{2}, 2 \gamma_{0}-c\right), \quad\left(2 \gamma_{0}-c, \tilde{c}^{+}\right) .
$$

In each of these intervals, the fraction at $U(\gamma)$ in the integrand in (38) is sign-definite and the function $U(\gamma)$ is continuous. This is why by the mean value theorem [4] we get

$$
L_{j}\left(\gamma_{0}\right)=U\left(\xi_{j}\right) \tilde{K}_{j}\left(\gamma_{0}\right), \quad j=1,3,4,5,
$$

here $\xi_{j}$ is a point in the integration interval $L_{j}\left(\gamma_{0}\right)$.

For $\tilde{K}_{1}\left(\gamma_{0}\right), \tilde{K}_{5}\left(\gamma_{0}\right)$, there hold the formulae obtained from (31), (17) via replacing $K$ and $\beta$ respectively by $\tilde{K}$ and $\tilde{\beta}$ and this is why by 40 we obtain $L_{1}\left(\gamma_{0}\right)=O\left(\gamma_{0}-c\right), L_{5}\left(\gamma_{0}\right)=$ $O\left(1 /\left(\operatorname{ls} \frac{\gamma_{0}-c}{2}\right)^{\tilde{\beta}}\right), \gamma_{0} \rightarrow c$. 
The integrals $\tilde{K}_{3}\left(\gamma_{0}\right), \tilde{K}_{4}\left(\gamma_{0}\right)$ satisfy the representations obtained from (26), (15) by the aforementioned replacement. This is why, taking into consideration that in formula (40) we have $U\left(\xi_{j}\right) \rightarrow U(c-0)$ for $j=3$ and $U\left(\xi_{j}\right) \rightarrow U(c+0)$ for $j=4$ as $\gamma_{0} \rightarrow c$, we get

$$
\begin{aligned}
& L_{3}\left(\gamma_{0}\right)=\frac{-U(c-0)}{\tilde{\beta}\left(\operatorname{ls} \frac{\gamma_{0}-c}{2}\right)^{\tilde{\beta}-1}}+o\left(1 /\left(\operatorname{ls} \frac{\gamma_{0}-c}{2}\right)^{\tilde{\beta}-1}\right)+O\left(1 /\left(\operatorname{ls} \frac{\gamma_{0}-c}{2}\right)^{\tilde{\beta}}\right), \quad \gamma_{0} \rightarrow c, \\
& L_{4}\left(\gamma_{0}\right)=\frac{U(c+0)}{\tilde{\beta}\left(\operatorname{ls} \frac{\gamma_{0}-c}{2}\right)^{\tilde{\beta}-1}}+o\left(1 /\left(\operatorname{ls} \frac{\gamma_{0}-c}{2}\right)^{\tilde{\beta}-1}\right)+O\left(1 /\left(\operatorname{ls} \frac{\gamma_{0}-c}{2}\right)^{\tilde{\beta}}\right), \quad \gamma_{0} \rightarrow c .
\end{aligned}
$$

Here, as usually, $o(\alpha)$ stands for an infinitesimal of order higher than the infinitesimal $\alpha$.

Denoting

$$
L_{6}^{\gamma_{0}}(c)=\int_{2 \gamma_{0}-c}^{\tilde{c}^{+}} \frac{U(\gamma)}{\left(\operatorname{ls} \frac{\gamma-c}{2}\right)^{\tilde{\beta}} \tan \frac{\gamma-c}{2}} d \gamma
$$

we consider the difference

$$
L_{6}\left(\gamma_{0}\right)-L_{6}^{\gamma_{0}}(c)=\int_{2 \gamma_{0}-c}^{\tilde{c}^{+}} \frac{U(\gamma)}{\left(\operatorname{ls} \frac{\gamma-c}{2}\right)^{\tilde{\beta}+1} \tan \frac{\gamma-c}{2}}\left(\operatorname{ls} \frac{\gamma-\gamma_{0}}{2}-\operatorname{ls} \frac{\gamma-c}{2}\right) d \gamma
$$

Since $\operatorname{ls} \frac{\gamma-\gamma_{0}}{2}-\operatorname{ls} \frac{\gamma-c}{2}>0$, by the mean value theorem we have

$$
\begin{aligned}
& L_{6}\left(\gamma_{0}\right)-L_{6}^{\gamma_{0}}(c)=U\left(\xi_{6}\right) \int_{2 \gamma_{0}-c}^{\tilde{c}^{+}} \frac{1}{\left(\operatorname{ls} \frac{\gamma-c}{2}\right)^{\tilde{\beta}-1} \tan \frac{\gamma-c}{2}}\left(\operatorname{ls} \frac{\gamma-\gamma_{0}}{2}-\operatorname{ls} \frac{\gamma-c}{2}\right) d \gamma, \\
& 2 \gamma_{0}-c \leqslant \xi_{6} \leqslant \tilde{c}^{+}
\end{aligned}
$$

or

$$
\begin{aligned}
L_{6}\left(\gamma_{0}\right)-L_{6}^{\gamma_{0}}(c)=U\left(\xi_{6}\right) \int_{2 \gamma_{0}-c}^{\tilde{c}^{+}}[ & -\tilde{A}\left(\gamma, \gamma_{0}, c\right) \operatorname{ls} \frac{\gamma-\gamma_{0}}{2} \\
& \left.+\frac{\operatorname{ls} \frac{\gamma-\gamma_{0}}{2}}{\left(\operatorname{ls} \frac{\gamma-\gamma_{0}}{2}\right)^{\tilde{\beta}+1} \tan \frac{\gamma-\gamma_{0}}{2}}-\frac{\operatorname{ls} \frac{\gamma-c}{2}}{\left(\operatorname{ls} \frac{\gamma-c}{2}\right)^{\tilde{\beta}+1} \tan \frac{\gamma-c}{2}}\right] d \gamma,
\end{aligned}
$$

where $\tilde{A}\left(\gamma, \gamma_{0}, c\right)$ is defined by the formula obtained from $(20)$ via replacing $A$ and $\beta$ respectively by $\tilde{A}$ and $\tilde{\beta}$. Calculating the integrals of two latter terms of the integrand, we obtain

$$
\begin{aligned}
L_{6}\left(\gamma_{0}\right)-L_{6}^{\gamma_{0}}(c)=U\left(\xi_{6}\right)\left\{\int_{2 \gamma_{0}-c}^{\tilde{c}^{+}}-\tilde{A}\left(\gamma, \gamma_{0}, c\right) \operatorname{ls} \frac{\gamma-\gamma_{0}}{2} d \gamma\right. \\
\left.+\left.\frac{1}{(\tilde{\beta}-1)\left(\operatorname{ls} \frac{\gamma-\gamma_{0}}{2}\right)^{\tilde{\beta}-1}}\right|_{2 \gamma_{0}-c} ^{\tilde{c}^{+}}-\left.\frac{1}{(\tilde{\beta}-1)\left(\operatorname{ls} \frac{\gamma-c}{2}\right)^{\tilde{\beta}-1}}\right|_{2 \gamma_{0}-c} ^{\tilde{c}^{+}}\right\} .
\end{aligned}
$$

Replacing $A, c^{+}, \beta$ by $\tilde{A}, \tilde{c}^{+}, \tilde{\beta}$, respectively, in formulae (19), 20, 24) we get

$$
\int_{2 \gamma_{0}-c}^{\tilde{c}^{+}}-\tilde{A}\left(\gamma, \gamma_{0}, c\right) \operatorname{ls} \frac{\gamma-\gamma_{0}}{2} d \gamma=O\left(1 /\left(\operatorname{ls} \frac{\gamma_{0}-c}{2}\right)^{\tilde{\beta}}\right), \quad \gamma_{0} \rightarrow c .
$$


The difference of two latter fractions in the right hand side in formula (42) as $\gamma=\tilde{c}^{+}$is an infinitesimal of order $\left(\gamma_{0}-c\right)$ as $\gamma_{0} \rightarrow c$, while as $\gamma=2 \gamma_{0}-c$ it is $O\left(1 /\left(\operatorname{ls} \frac{\gamma_{0}-c}{2}\right)^{\tilde{\beta}}\right)$ by formula (30) with $\beta=\tilde{\beta}$.

Thus, formula 42 ) shows that

$$
L_{6}\left(\gamma_{0}\right)-L_{6}^{\gamma_{0}}(c)=O\left(1 /\left(\operatorname{ls} \frac{\gamma_{0}-c}{2}\right)^{\tilde{\beta}}\right), \quad \gamma_{0} \rightarrow c .
$$

Since $\tilde{\beta}>1$, the integral

$$
L_{6}(c)=\int_{c}^{\tilde{c}^{+}} \frac{U(\gamma)}{\left(\operatorname{ls} \frac{\gamma-c}{2}\right)^{\tilde{\beta}} \tan \frac{\gamma-c}{2}} d \gamma
$$

is well-defined [4] since $U(\gamma)$ is a continuous in the interval $\left[c, \tilde{c}^{+}\right]$function, and the second factor in the integrand is a positive integrable function.

Employing the mean value theorem, we write the difference $L_{6}(c)-L_{6}^{\gamma_{0}}(c)$ being an integral over the interval $\left(c, 2 \gamma_{0}-c\right)$ as

$$
\begin{aligned}
& L_{6}(c)-L_{6}^{\gamma_{0}}(c) \\
& =\left.U\left(\xi_{6}^{*}\right) \frac{1}{(\tilde{\beta}-1)\left(\operatorname{ls} \frac{\gamma-c}{2}\right)^{\tilde{\beta}-1}}\right|_{c} ^{2 \gamma_{0}-c}=\frac{U\left(\xi_{6}^{*}\right)}{(\tilde{\beta}-1)\left(\operatorname{ls}\left(\gamma_{0}-c\right)\right)^{\tilde{\beta}-1}}, \quad c \leqslant \xi_{6}^{*} \leqslant 2 \gamma_{0}-c .
\end{aligned}
$$

By (30) this implies

$$
L_{6}(c)-L_{6}^{\gamma_{0}}(c)=\frac{U(c+0)}{(\tilde{\beta}-1)\left(\operatorname{ls} \frac{\gamma_{0}-c}{2}\right)^{\tilde{\beta}-1}}+o\left(1 /\left(\operatorname{ls} \frac{\gamma_{0}-c}{2}\right)^{\tilde{\beta}-1}\right)+O\left(1 /\left(\operatorname{ls} \frac{\gamma_{0}-c}{2}\right)^{\tilde{\beta}}\right), \quad \gamma_{0} \rightarrow c .
$$

Now by (44), (46) we write

$$
L_{6}\left(\gamma_{0}\right)-L_{6}(c)=\frac{-U(c+0)}{(\tilde{\beta}-1)\left(\operatorname{ls} \frac{\gamma_{0}-c}{2}\right)^{\tilde{\beta}-1}}-o\left(1 /\left(\operatorname{ls} \frac{\gamma_{0}-c}{2}\right)^{\tilde{\beta}-1}\right)+O\left(1 /\left(\operatorname{ls} \frac{\gamma_{0}-c}{2}\right)^{\tilde{\beta}}\right), \quad \gamma_{0} \rightarrow c .
$$

We introduce the notation

$$
L_{2}^{\gamma_{0}}(c)=\int_{\tilde{c}_{\gamma_{0}}^{-}}^{2 c-\gamma_{0}} \frac{U(\gamma)}{\left(\operatorname{ls} \frac{\gamma-c}{2}\right)^{\tilde{\beta}} \tan \frac{\gamma-c}{2}} d \gamma
$$

and we consider the difference

$$
L_{2}\left(\gamma_{0}\right)-L_{2}^{\gamma_{0}}(c)=\int_{\tilde{c}_{\gamma_{0}}^{-}}^{2 c-\gamma_{0}} \frac{U(\gamma)}{\left(\operatorname{ls} \frac{\gamma-c}{2}\right)^{\tilde{\beta}+1} \tan \frac{\gamma-c}{2}}\left(\operatorname{ls} \frac{\gamma-\gamma_{0}}{2}-\operatorname{ls} \frac{\gamma-c}{2}\right) d \gamma
$$

Taking into consideration that

$$
\operatorname{ls} \frac{\gamma-\gamma_{0}}{2}-\operatorname{ls} \frac{\gamma-c}{2}<0,
$$

by the mean value theorem we obtain

$$
L_{2}\left(\gamma_{0}\right)-L_{2}^{\gamma_{0}}(c)=U\left(\xi_{2}\right) \int_{\tilde{c}_{\gamma_{0}}^{-}}^{2 c-\gamma_{0}} \frac{\operatorname{ls} \frac{\gamma-\gamma_{0}}{2}-\operatorname{ls} \frac{\gamma-c}{2}}{\left(\operatorname{ls} \frac{\gamma-c}{2}\right)^{\tilde{\beta}+1} \tan \frac{\gamma-c}{2}} d \gamma, \quad \tilde{c}_{\gamma_{0}}^{-} \leqslant \xi_{2} \leqslant 2 c-\gamma_{0} .
$$


Proceeding as above, we write this formula as

$$
\begin{aligned}
& L_{2}\left(\gamma_{0}\right)-L_{2}^{\gamma_{0}}(c) \\
& =U\left(\xi_{2}\right) \cdot \int_{\tilde{c}_{\gamma_{0}}^{-}}^{2 c-\gamma_{0}}\left[-\tilde{A}\left(\gamma, \gamma_{0}, c\right) \operatorname{ls} \frac{\gamma-\gamma_{0}}{2} d \gamma+\frac{1}{\left(\operatorname{ls} \frac{\gamma-c}{2}\right)^{\tilde{\beta}} \tan \frac{\gamma-\gamma_{0}}{2}}-\frac{1}{\left(\operatorname{ls} \frac{\gamma-c}{2}\right)^{\tilde{\beta}} \tan \frac{\gamma-c}{2}}\right] d \gamma
\end{aligned}
$$

where $\tilde{A}\left(\gamma, \gamma_{0}, c\right)$ stands for the same as in formula (41). Hence, similar to formula 42, we get

$$
\begin{aligned}
L_{2}\left(\gamma_{0}\right)-L_{2}^{\gamma_{0}}(c)=U\left(\xi_{2}\right) \cdot\{ & \int_{\tilde{c}_{\gamma_{0}}^{-}}^{2 c-\gamma_{0}}-\tilde{A}\left(\gamma, \gamma_{0}, c\right) \operatorname{ls} \frac{\gamma-\gamma_{0}}{2} d \gamma+\left.\frac{1}{(\tilde{\beta}-1)\left(\operatorname{ls} \frac{\gamma-\gamma_{0}}{2}\right)^{\tilde{\beta}-1}}\right|_{\tilde{c}_{\gamma_{0}}^{-}} ^{2 c-\gamma_{0}}- \\
& \left.-\left.\frac{1}{(\tilde{\beta}-1)\left(\operatorname{ls} \frac{\gamma-c}{2}\right)^{\tilde{\beta}-1}}\right|_{\tilde{c}_{\gamma_{0}}^{-}} ^{2 c-\gamma_{0}}\right\} .
\end{aligned}
$$

On base of the formulae obtained from (20), (28), (29) by the aforementioned replacement, for the integral in the right hand side in formula $(49)$ we obtain a relation similar to (43); the latter difference in the right hand side of formula (49) is estimated in the same way as the corresponding difference in formula $(42)$. Thus, we arrive at the relation

$$
L_{2}\left(\gamma_{0}\right)-L_{2}^{\gamma_{0}}(c)=O\left(1 /\left(\operatorname{ls} \frac{\gamma_{0}-c}{2}\right)^{\tilde{\beta}}\right), \quad \gamma_{0} \rightarrow c .
$$

Since $\tilde{\beta}>1$, the integral

$$
L_{2}(c)=\int_{\tilde{c}^{-}}^{c} \frac{U(\gamma)}{\left(\operatorname{ls} \frac{\gamma-c}{2}\right)^{\tilde{\beta}} \tan \frac{\gamma-c}{2}} d \gamma
$$

similar to the integral $L_{6}(c)$ in formula (45) is well-defined and according (38), the sum

$$
L(c)=L_{2}(c)+L_{6}(c)
$$

is well-defined.

By (47), (51) we have

$$
L_{2}(c)-L_{2}^{\gamma_{0}}(c)=\left(\int_{\tilde{c}^{-}}^{\tilde{c}_{\gamma_{0}}^{-}}+\int_{2 c-\gamma_{0}}^{c}\right) \frac{U(\gamma)}{\left(\operatorname{ls} \frac{\gamma-c}{2}\right)^{\tilde{\beta}} \tan \frac{\gamma-c}{2}} d \gamma
$$

Here we write the integrals in the right hand side by employing the mean value theorem similar to formula 48). Calculating the remaining integrals, we get

$$
\begin{aligned}
& L_{2}(c)-L_{2}^{\gamma_{0}}(c)=\left.U\left(\xi_{11}\right) \frac{1}{(\tilde{\beta}-1)\left(\operatorname{ls} \frac{\gamma-c}{2}\right)^{\tilde{\beta}-1}}\right|_{\tilde{c}^{-}} ^{\tilde{c}_{\gamma_{0}}^{-}}-U\left(\xi_{31}\right) \frac{1}{(\tilde{\beta}-1)\left(\operatorname{ls} \frac{\gamma_{0}-c}{2}\right)^{\tilde{\beta}-1}}, \\
& \tilde{c}^{-} \leqslant \xi_{11} \leqslant \tilde{c}_{\gamma_{0}}^{-}, \quad 2 c-\gamma_{0} \leqslant \xi_{31} \leqslant c .
\end{aligned}
$$

The first term in the right hand side of the latter formula is an infinitesimal of order $\left(\gamma_{0}-c\right)$ as $\gamma_{0} \rightarrow c$. In the second term $U\left(\xi_{31}\right) \rightarrow U(c-0)$ as $\gamma_{0} \rightarrow c$. This is why

$$
L_{2}(c)-L_{2}^{\gamma_{0}}(c)=\frac{-U(c-0)}{(\tilde{\beta}-1)\left(\operatorname{ls} \frac{\gamma_{0}-c}{2}\right)^{\tilde{\beta}-1}}+o\left(1 /\left(\operatorname{ls} \frac{\gamma_{0}-c}{2}\right)^{\tilde{\beta}-1}\right), \quad \gamma_{0} \rightarrow c .
$$


In view of formula (50), this implies

$$
L_{2}\left(\gamma_{0}\right)-L_{2}^{\gamma_{0}}(c)=\frac{U(c-0)}{(\tilde{\beta}-1)\left(\operatorname{ls} \frac{\gamma_{0}-c}{2}\right)^{\tilde{\beta}-1}}-o\left(1 /\left(\operatorname{ls} \frac{\gamma_{0}-c}{2}\right)^{\tilde{\beta}-1}\right)+O\left(1 /\left(\operatorname{ls} \frac{\gamma_{0}-c}{2}\right)^{\tilde{\beta}}\right), \quad \gamma_{0} \rightarrow c .
$$

On the base of formula $(39)$ and subsequent relations we get

$$
\begin{aligned}
L\left(\gamma_{0}\right)= & L(c)-\frac{1}{\tilde{\beta}(\tilde{\beta}-1)} \cdot(U(c+0)-U(c-0)) \cdot \frac{1}{\left(\operatorname{ls} \frac{\gamma_{0}-c}{2}\right)^{\tilde{\beta}-1}} \\
& +o\left(1 /\left(\operatorname{ls} \frac{\gamma_{0}-c}{2}\right)^{\tilde{\beta}-1}\right)+O\left(1 /\left(\operatorname{ls} \frac{\gamma_{0}-c}{2}\right)^{\tilde{\beta}}\right), \quad \gamma_{0} \rightarrow c .
\end{aligned}
$$

We substitute expression (37) into formula (36), then in the right hand side of the obtained relation, thanks to the differentiability, each term except $L\left(\gamma_{0}\right)$ can be represented as the sum of its value at the point $c$ and an infinitesimal of order $\left(\gamma_{0}-c\right)$ as $\gamma_{0} \rightarrow c$. Observing that the obtained relation holds also for $\gamma_{0}=c$, we arrive at the identity

$$
J_{3}\left(\gamma_{0}\right)-J_{3}(c)=L\left(\gamma_{0}\right)-L(c)+O\left(\gamma_{0}-c\right), \quad \gamma_{0} \rightarrow c .
$$

Formulae (52), (53) give a representation for $J_{3}\left(\gamma_{0}\right)$.

Thanks to relations (34), (35), (52), (53) and in view of formula (6) implying $I(c)=\sum_{j=1}^{4} J_{j}(c)$, we arrive at the following representation

$$
\begin{aligned}
I\left(\gamma_{0}\right)-I(c)= & \frac{\Phi(c-0)-\Phi(c+0)}{(\beta-1)\left(-\ln \sin ^{2} \frac{\gamma_{0}-c}{2}\right)^{\beta-1}}-\frac{U(c+0)-U(c-0)}{\tilde{\beta}(\tilde{\beta}-1)\left(-\ln \sin ^{2} \frac{\gamma_{0}-c}{2}\right)^{\tilde{\beta}-1}} \\
& +o\left(1 /\left(-\ln \sin ^{2} \frac{\gamma_{0}-c}{2}\right)^{\tilde{\beta}-1}\right)+O\left(1 /\left(-\ln \sin ^{2} \frac{\gamma_{0}-c}{2}\right)^{\beta}\right)
\end{aligned}
$$

as $\gamma_{0} \rightarrow c$ with $\tilde{\beta}=\beta+\delta, \beta>1, \delta>0$. In particular, this yields that the integral $I\left(\gamma_{0}\right)$ is a function continuous at the point $c$.

Making appropriate changes in the above formulae and in arguing, it is easy to check that this representation is also true for values $\gamma_{0}<c$ close to $c$.

It is reasonable to note that in formula (54),

$$
U(c+0)-U(c-0)=\tilde{\beta}(\chi(c+0)-\chi(c-0)),
$$

since $U(\gamma)=\nu(\gamma)+\tilde{\beta} \underset{\tilde{\beta}}{\chi}(\gamma), \nu(c \pm 0)=0$.

As $\beta=1$, we have $\tilde{\beta}=\beta+\delta>1$ and representations (52), (53) and relations (35) are still true. Making obvious changes in the above formulae for $J_{4}\left(\gamma_{0}\right)$, we arrive at the representation

$$
\begin{aligned}
I\left(\gamma_{0}\right)= & J_{1}(c)+J_{2}(c)+[\Phi(c+0)-\Phi(c-0)] \ln \left(-\ln \sin ^{2} \frac{\gamma_{0}-c}{2}\right)+N \\
& -\frac{U(c+0)-U(c-0)}{\tilde{\beta}(\tilde{\beta}-1)} \cdot \frac{1}{\left(-\ln \sin ^{2} \frac{\gamma_{0}-c}{2}\right)^{\tilde{\beta}-1}} \\
& +o\left(1 /\left(-\ln \sin ^{2} \frac{\gamma_{0}-c}{2}\right)^{\tilde{\beta}-1}\right)+O\left(1 /\left(-\ln \sin ^{2} \frac{\gamma_{0}-c}{2}\right)\right), \quad \gamma_{0} \rightarrow c,
\end{aligned}
$$

where $N=[\Phi(c-0)-\Phi(c+0)] \ln \left(-\ln \sin ^{2} e^{-2}\right)$.

\section{Conclusion}

Thus, we arrive at the following statement. 
Theorem 1. If the density in integral (1) obeys formula (5), in which $\chi(\gamma)$ is a function with the derivative of form (4), then integral (1) satisfies representation (54) as $\beta>1$ and representation (55) as $\beta=1$.

Remark 1. If the function $\nu(\gamma)$ in formula (4) satisfies the representation

$$
\nu(\gamma)=\frac{\nu_{1}(\gamma)}{\left(\operatorname{ls} \frac{\gamma-c}{2}\right)^{\varkappa}}, \quad \varkappa=\text { const }>0, \quad \gamma \in\left[\tilde{c}^{-}, \tilde{c}^{+}\right]
$$

where $\nu_{1}(\gamma)$ is a function continuous in each of the intervals $\left[\tilde{c}^{-}, c\right],\left[c, \tilde{c}^{+}\right]$and $\nu_{1}(c)=0$, the statement of the theorem can be specified by replacing the term o $\left(1 /\left(\mathrm{ls}_{\mathrm{s}} \frac{\gamma_{0}-c}{2}\right)^{\tilde{\beta}-1}\right)$ by $O\left(1 /\left(\operatorname{ls} \frac{\gamma_{0}-c}{2}\right)^{\tilde{\beta}-1+\varkappa}\right)$.

Indeed, in this case by (4) we have

$$
\chi^{\prime}(\gamma)=\nu_{1}(\gamma) /\left(\left(\operatorname{ls} \frac{\gamma-c}{2}\right)^{\varkappa+1} \tan \frac{\gamma-c}{2}\right) .
$$

Applying the mean value theorem, for $\gamma>c$ we obtain

$$
\chi(\gamma)-\chi(c+0)=\int_{c}^{\gamma} \chi^{\prime}(\gamma) d \gamma=\frac{1}{\varkappa} \frac{\nu_{1}(\eta)}{\left(\operatorname{ls} \frac{\gamma-c}{2}\right)^{\varkappa}}, \quad \eta \in[c, \gamma]
$$

at that, for $\gamma>c$ we represent the function $U(\gamma)=\nu(\gamma)+\tilde{\beta} \chi(\gamma)$ in formula (38) as

$$
U(\gamma)=U(c+0)+\frac{\nu_{1}(\gamma)+\frac{\tilde{\beta}}{\varkappa} \nu_{1}(\eta)}{\left(\operatorname{ls} \frac{\gamma-c}{2}\right)^{\varkappa}}
$$

Hence, denoting $M_{1}=\max _{c \leqslant \gamma \leqslant \tilde{c}^{+}}\left|\nu_{1}(\gamma)\right|$, we get

$$
|U(\gamma)-U(c+0)| \leqslant M_{1}\left(1+\frac{\tilde{\beta}}{\varkappa}\right) /\left(\operatorname{ls} \frac{\gamma-c}{2}\right)^{\varkappa}
$$

and for $\xi \in(c, \gamma)$ we obtain

$$
|U(\xi)-U(c+0)| \leqslant M_{1}\left(1+\frac{\tilde{\beta}}{\varkappa}\right) /\left(\operatorname{ls} \frac{\xi-c}{2}\right)^{\varkappa} \leqslant M_{1}\left(1+\frac{\tilde{\beta}}{\varkappa}\right) /\left(\operatorname{ls} \frac{\gamma-c}{2}\right)^{\varkappa} .
$$

In the case $\gamma<c, \gamma \leqslant \xi \leqslant c$ we arrive at the same inequality.

These inequalities justifies the aforementioned change in the formulae for $L_{j}\left(\gamma_{0}\right), j=2,3,4,6$ and in representations (52), (54).

The results of the present paper describe the behavior of integral (1) in the vicinity of a point of weak singularity of its density. They are close to known results by N.I. Muskhelishvili on the behavior of Cauchy type integral (and of singular integral) in the vicinity of a point of power type singularity of the density [3] and they can be employed while studying the properties of the solutions to various boundary value problems for analytic functions.

Rather weak restrictions imposed for the density in integral (1) in the present paper give an opportunity to apply the results for studying the behavior of a conformal mapping near an angular point at the boundary of a domain corresponding to a canonical domain with a smooth boundary.

Papers [5], [6] and aforementioned papers [1], [2] belong to the same scientific field as the present paper and they can be of interest for the reader. 


\section{BIBLIOGRAPHY}

1. R.B. Salimov. Solution of a singular homogeneous Hilbert boundary-value problem for analytic function in multiply connected circular domain // Izv. VUZov. Matem. 6, 37-44 (2013). [Russ. Math. 58:5, 63-66 (2014).]

2. R.B. Salimov, Yu.A. Shamgin. Study of behavior of singular integral with Hilbert kernel at point of weak continuity of density // Trudy Matem. Centra im. N.I. Lobachevskogo. 46, 399-402 (2013).

3. N.I. Muskhelishvili. Singular integral equations. Nauka, Moscow (1968). [Dover Publications, New York (1992).]

4. G.M. Fikhtengol'ts. Course of differential and integral calculus. V. 2. Nauka, Moscow (1970). (in Russian).

5. R.B. Salimov. The behavior of a singular integral with the Hilbert kernel near a point where the density of the integral is weakly continuous // Izv. VUZov. Matem. 6, 61-66 (2012). [Russ. Math. 56:6, 52-56 (2012).]

6. R.B. Salimov. New asymptotic representation of a singular integral with the Hilbert kernel near a point of weak continuity of its density // Izv. VUZov. Matem. 4, 73-78 (2016). [Russ. Math. 60:4, 60-64 (2016).]

Rasikh Bakhtigareevich Salimov,

Kazan State University of Architecture and Engineering,

Zelenaya str. 1,

420043, Kazan, Russia

E-mail: salimov.rsb@gmail.com 